\title{
Guidelines for COVID-19 Epidemiological Investigations
}

\author{
Chinese Center for Disease Control and Prevention
}

These guidelines are based on the most recent research on the epidemiology of COVID-19 from home and abroad and are intended to further improve epidemiological investigations of COVID-19 cases to control spread of the virus.

\section{PURPOSE}

1. To investigate source of infection and trace and identify close contacts;

2. To investigate onset of illness and treatment of patients, including clinical characteristics and risk factors;

3. To investigate and analyze transmission characteristics of COVID-19 cases and clusters of cases.

\section{SCOPE}

The guidelines cover investigation of COVID-19 suspected cases, confirmed cases, asymptomatic infected persons, and clusters of cases.

\section{CONTENT AND METHODS}

\section{Case Investigation}

The county (district) level disease control and prevention agency, upon receiving a report of one or more COVID-19 cases, should complete an initial epidemiological investigation within 24 hours. The investigation can be conducted through review of documents and interviews with patients, physicians, acquaintances, and others as needed. When a patient has completed a 14-day period of isolation and health monitoring, the county (district) level disease control and prevention agency should obtain information on specimen collection and testing for inclusion in the case report.

Case investigation should include basic demographic and identification data and information on onset of symptoms, care-seeking behavior, risk factors, potential exposures, and laboratory findings of suspected cases, confirmed cases, and asymptomatic infected persons. For suspected cases, this information is to be recorded in a Case Investigation Questionnaire (Appendix S1, available in http://weekly.chinacdc.cn/) that includes identifying information such as name, gender, and ID number. Information about individuals identified as close contacts should be recorded in the Health Status Monitoring Case Form for Close Contacts in the Guideline for Investigation and Management of Close Contacts of COVID-19 Cases.

\section{Investigations on Clusters of Cases}

A cluster of cases is the detection of 2 or more confirmed cases or asymptomatic infected persons in a small area (e.g., in a family, an office, a school class, or workplace) within a 14-day period and that has the possibility of person-to-person transmission or a common exposure.

Based on case reports in the Infectious Disease Reporting Information Management System (IDRIMS) and case investigation findings potentially meeting the above definition, the county (district) level disease control and prevention agency should immediately launch an investigation into a possible cluster of cases. Investigations should include information on source of infection and close contacts. The investigation should focus on epidemiological links among cases; chains of transmission and transmission routes should be analyzed. For investigation and analytic methods, refer to Appendix S2. (available in http://weekly.chinacdc.cn/)

\section{ORGANIZATION AND IMPLEMENTATION}

Using the principle of "localized management," health authorities of the county or district where the individual being investigated sought medical care shall have the disease control and prevention agency conduct an epidemiological investigation of the case or cluster of cases. The investigating agency should promptly establish an investigation team, clarify the purpose of the investigation, develop an investigation plan, select team members, and assign duties and tasks. During all investigations, investigators should use proper personal protective equipment. Professionals from disease control and prevention agencies at the 
prefecture, provincial, and national levels should accompany county or district level teams when requested or when deemed necessary.

\section{ANALYSIS AND REPORTING}

Upon completion of an investigation of confirmed cases, asymptomatic infected persons, or cluster of cases, the county (district) level disease control and prevention agency should submit a case investigation form or investigation report to the online reporting system. The local CDC should review the submitted report and update it in a timely manner as the investigation progresses.

For investigations of clusters of cases, all basic information and all initial, interim, and final reports should be submitted in accordance with the National Protocol for Reporting and Management of Public Health Emergency Related Information (Trial). All key information of these investigations (see Appendix S2) should be included in the final report.

Acknowledgement: China CDC Weekly would like to thank Xijuan $\mathrm{Fu}$ of the WHO China Office, Canjun Zheng, Liping Wang, Zhaorui
Chang, Zhongjie Li, Lance Rodewald, and Huilai Ma of China CDC for their contributions in translating and editing this document (1).

The Chinese document written by experts in China CDC Strategy and Policy Working Group for COVID-19 Emergency Response, including Zijian Feng of China CDC, Canjun Zheng, Zhaorui Chang, Fengfeng Liu, Mengjie Geng, Junling Sun, Sheng Zhou, Lu Ran, Huilai Ma, Liping Wang, Zhongjie Li of Division of Infectious Disease, Key Laboratory of Surveillance and Early Warning on Infectious Disease, China CDC, Hui Chen of National Center for Tuberculosis Control and Prevention, China CDC, Jingwei Jiang of National Institute for Communicable Disease Control and Prevention, China CDC, and Jian $\mathrm{Li}$ of National Center for AIDS Control and Prevention, China CDC.

doi: $10.46234 / \mathrm{ccdcw} 2020.083$

Submitted: March 14, 2020; Accepted: March 17, 2020

\section{REFERENCES}

\footnotetext{
1. Chinese Center for Disease Control and Prevention. Technical documents for COVID-19 prevention and control. http://www. chinacdc.cn/jkzt/crb/zl/szkb_11803/jszl_11815/202003/t20200309_214 241.html. [2020-3-9]. (In Chinese).
} 


\section{Appendix S1}

\section{Investigation Questionnaire for COVID-19 Cases}

Questionnaire number: ID number:

\section{Basic information}

1. Name:

2. Sex: $\square$ Male $\square$ Female

3. Imported case from outside China: $\square$ Yes $\square$ No

If yes, please fill in the following:

Country(ies) and region(s) of residence or travel before entry (multiple answers possible):

Country(ies) or region(s) where transfer(s) were made before entry:

Nationality: Passport Number:

Point of entry: of Province (e.g., airport, train station, or wharf)

Date of entry: (dd) $/(\mathrm{mm}) /$ (yyy)

Transportation (e.g., flight, train, or ship number):

\section{Onset of symptoms and care-seeking behavior}

4. How was the individual identified:

$\square$ voluntary care seeking $\square$ via close contact management

$\square$ at border screening $\square$ through active population screening

$\square$ through routine surveillance of influenza-like illness or SARI $\square$ other

5. Date of hospital admission: $(\mathrm{dd}) /(\mathrm{mm}) /($ yyyy $)$

6. Symptoms and signs at admission:

Fever: maximum temperature ${ }^{\circ} \mathrm{C}$

$\square$ chills $\square$ dry cough $\square$ sputum $\square$ nasal congestion $\square$ rhinorrhea $\square$ sore throat

headache $\square$ fatigue $\square$ muscle soreness $\square$ arthralgia

$\square$ shortness of breath $\square$ dyspnea $\square$ chest tightness $\square$ chest pain $\square$ conjunctival congestion $\square$ nausea vomiting $\square$ diarrhea $\square$ abdominal pain $\square$ other

7. Are there any medical complications? $\square$ Yes $\square$ No

If yes, please select (more than one selection is possible): $\square$ meningitis $\square$ encephalitis $\square$ bacteremia/sepsis

$\square$ myocarditis $\square$ acute lung injury/ARDS $\square$ acute kidney injury $\square$ epilepsy

$\square$ secondary bacterial pneumonia $\square$ other

8. Chest X-ray/CT consistent with pneumonia: $\square$ Not performed $\square$ No $\square$ Yes

9. Date of discharge: $(\mathrm{dd}) /(\mathrm{mm}) /($ yyyy $)$

\section{Risk factors and exposure history}

10. Is the patient in any of these occupational groups:

$\square$ health care worker $\square$ microbiologist $\square$ not from a specific occupational group

$\square$ personnel with wildlife contact $\square$ poultry and livestock farming workers $\square$ other

If yes for health care worker, please select: $\square$ doctor $\square$ nurse $\square$ field staff for disease control and prevention laboratory staff $\square$ other 
11. Is/was the patient pregnant: $\square$ Yes, pregnant for weeks $\square$ no

12. Past medical history (multiple selections possible): $\square$ none $\square$ hypertension $\square$ diabetes $\square$ cardiovascular and cerebrovascular diseases $\square$ asthma $\square$ chronic pulmonary ( $\square$ chronic obstructive pulmonary disease, $\square$ other___ $) \square$ cancer ( $\square$ lung cancer $\square$ other__ $\quad \square$ chronic kidney disease $\square$ chronic liver disease immunodeficiency diseases $\square$ postpartum (within 6 weeks) $\square$ other

13. Lived in Wuhan and surrounding areas, or other domestic communities with reported cases:

$\square$ travel history $\square$ residence history $\square$ none

If yes, please fill in: ___ county/district __ prefecture ___ province

14. Has the patient visited or lived in overseas country(ies) or region(s) with outbreaks:

$\square$ travel history $\square$ residence history $\square$ no

If yes, please specify country(ies) or region(s):

15. Has the patient come in contact with a person who has fever and/or respiratory symptoms from Wuhan and surrounding areas or from a domestic community with a reported case/cases:

Yes $\square$ No $\square$ Unclear

16. Has the patient come in contact with a person who has fever and/or respiratory symptoms from overseas country(ies) or region(s) with outbreaks:

$\square$ Yes $\square$ No $\square$ Unclear

17. Has the patient come in contact with a confirmed case or an asymptomatic infected person:

$\square$ Yes $\square$ No $\square$ Unclear

18. Has the patient been involved in a cluster of cases in the same family, office, school, kindergarten or nursery, or workshop? $\square$ Yes $\square$ No $\square$ Unclear

\section{Laboratory testing}

Record times and results of specimens collected and tested on initial sampling and every sample until the last sample collected during isolation until discharge (include positive and negative results for each specimen)

\begin{tabular}{|l|l|l|}
\hline \multicolumn{1}{|c|}{ Specimen type } & Sampling time (dd/mm/yyyy) & Test result(+/- /to be tested) \\
\hline Throat swab & & \\
\hline Nasal swab & & \\
\hline Nasopharyngeal swab & & \\
\hline Sputum & & \\
\hline Tracheal secretions & & \\
\hline Tracheal aspirate & & \\
\hline Alveolar lavage fluid & & \\
\hline Urine & & \\
\hline Stool/rectal swab & & \\
\hline Blood (nucleic acid testing) & & \\
\hline Blood (IgM) & & \\
\hline Blood (lgG) & & \\
\hline Blood (IgM+lgG) & & \\
\hline $\begin{array}{l}\text { Blood, with IgG increase of 4 times or more from initial to } \\
\text { convalescent sample* }\end{array}$ & & \\
\hline Other (specimen type) & & \\
\hline None collected (do not fill in the sampling time or results) & & \\
\hline
\end{tabular}

*: If IgG in convalescent blood increased by 4 times over the initial sample, the test result is positive.

Investigating organization: Investigator signature:

Investigation date: (dd)/(mm)/(yyyy) 


\section{Appendix S2}

\section{Investigation and Analytic Methods for Clusters of COVID-19 Cases}

\section{Definition of cluster of cases}

A cluster of cases refers to the detection of 2 or more confirmed cases or asymptomatic infected persons in a small area (e.g., in a family, an office, a school class, or workplace) within a 14-day period and that has the possibility of person-to-person transmission or a common exposure.

\section{Detection}

1. Through case investigation, find confirmed cases, suspected cases, and asymptomatic infected persons who have had close contact with a case or who may have had a common exposure with a case.

2. Through the Infectious Disease Reporting Information Management System (IDRIMS), search for confirmed cases, suspected cases, and asymptomatic infected persons working for the same employer, or those who have the same address and whose date of illness onset was within 1 or 2 incubation periods.

3. Through consolidating and reviewing case investigation reports, search for confirmed cases, suspected cases, and asymptomatic infected persons from different regions with the same exposure history, such as taking the same flight or train, or participation in a common travel group or attended a common conference within 14 days before onset of illness.

\section{Investigation content}

\section{Patients and close contacts}

The investigation of clusters of cases should focus on: (1) whether the patients and close contacts have a history of travel to or residence in Wuhan and its surrounding areas, other domestic communities with reported cases, or overseas countries or regions with severe outbreaks; (2) whether they have been in contact with patients with fever or respiratory symptoms from Wuhan and its surrounding areas, other domestic communities with reported cases, or overseas countries or regions with severe outbreaks; (3) the type of contact, contact distance, frequency of occurrence, and personal protective measures taken; (4.) case-related activity tracking; and (5) verification and registration of the name, ID number, and contact details of patients.

For the initial investigation, the time period for clusters of cases should not be limited to 14 days. The relevant suspected cases and asymptomatic infected persons should be included in the investigation. When the investigation is closed, a final determination should be made whether it was a cluster of cases based on epidemiological and laboratory findings.

The investigation of close contacts should focus on: (1) the onset of symptoms and specimen collection and testing of close contacts; (2) the types of close contacts, such as having meals together, living in the same household, sharing transportation, etc.; and (3) the health outcome of close contacts.

2. Place of exposure

(1) Family exposures: investigate the number of family members living together and their contacts and personal protection; the household environment, including number of rooms, floor area, ventilation and air conditioning use, and hand washing facilities; and elevator use and status of building disinfection.

(2) Mealtime exposures: investigate the time, place, attendees and seating arrangements, dining environment, ventilation and air conditioning use, hand washing facilities, and behaviors that may lead to increased risk of transmission.

(3) Business exposures: investigate the number of workers; the distribution of work stations; the distribution of workshops; the type of work contact and the protection of workers in the workplace; the environmental health of the workplace, canteen/cafeteria, dormitory, toilet, and other relevant places; use of central air conditioning, air cleaning or circulation systems, and ventilation; hand washing facilities; elevator use; and disinfection. 
(4) Means of transportation: investigate the means of transportation, seat distribution, ventilation and use of air conditioning, disinfection status, hand washing facilities, number of passengers, health conditions, and use of personal protective equipment.

(5) Public places: the length of stay in shopping malls, supermarkets, public baths, hotels, nursing homes, hospitals, wedding/funeral sites, and other public places where patients may have been exposed; number and density of people; personal protection; layout and floor area of public places; use of ventilation and air conditioning; use and disinfection of elevators; and hand washing facilities.

\section{Sampling and testing}

Specimen collection and testing should be done for all cases in accordance with relevant requirements. For special circumstances such as the index case of a cluster of cases, people suspected to be asymptomatically infected, or patients having transmitted during the incubation period, increase the frequency of sampling and testing. Use of two negative nucleic acid tests rather than relying on a single negative test, and collecting two serum specimens within 7 days and between 3-4 weeks after onset is recommended for future reference in these special circumstances.

\section{Information analysis}

\section{Analysis of transmission chains}

Draw epidemic curves according to the onset time of cases. Draw the onset time sequence diagram or case relationship diagram (see Figure S1) with information including the relation with the index case, the exposure history up to 14 days before onset and activities after onset of illness. Once completed, the transmission chain should be thoroughly analyzed in view of all available information.

\section{Analysis of Generation of Infection}

According to the epidemic curve, time sequence diagram, case relationship diagram, information of incubation period, and exposure history, determine the generation of each case. The following principles can be referred to in determining the generation of each case.

The first generation is usually the case with the earliest onset, i.e. the index case of a cluster of cases. If asymptomatic infected persons or transmission during the incubation period is suspected, comprehensive analysis and determination should be based on epidemiological investigation and laboratory testing results.

In principle, the following three criteria should be met for determining the second generation of cases: (1) history of contact with the first generation case within 14 days before onset of illness; (2) within 14 days before onset, the patient has never visited or lived in Wuhan and surrounding areas, in other domestic communities with reported cases, or in overseas countries or regions with severe outbreaks; (3) within 14 days before onset, there was no other suspected exposure history, such as visiting a hospital or a community with ongoing virus transmission.

These three criteria for determining the second generation of cases can also be used for identifying third generation cases. If the patient has been in contact with the previous two generations within 14 days before onset, the generation cannot be confirmed.

\section{Analysis of the incubation period}

The following three criteria should be met to accurately calculate the incubation period for a single case: (1) the second-generation case has a clear contact history with the index case; (2) the second-generation case has had at least some short contact time with the index case; (3) the second-generation case has no other exposure history or contact history before onset other than with the index case.

In a cluster outbreak, if the incubation period of a single case is found to have exceeded the minimal and maximal value observed in existing studies, verifying whether the above criteria are met and confirm the accuracy of the onset time of the patient's illness and the contact time with the index case.

\section{Analysis of infectivity during incubation}

In a cluster outbreak, if the index case is determined to have been infective during the incubation period, the following three criteria should be met: (1) the index case and the second-generation case did not have any clinical symptoms or signs of infection at the time of contact, and there was no contact history between these two cases after onset; (2) the onset of illness for the second-generation case happened within 14 days after the last contact with the 
index case; (3) the second-generation case had no other exposure history or contact history other than that with the index case.

It is recommended that sampling be done as early as possible for the index case during the investigation. If the sampling of the positive specimen of the index case is earlier than the onset of the second-generation case, the evidence is considered to be stronger. In addition, it is recommended to collect two serum specimens within 7 days and between 3-4 weeks after onset of illness of the index case for future reference.

\section{Analysis of infectivity of asymptomatic infected persons}

In a cluster of cases, if an asymptomatic infected person is determined to be the source of infection, the following three conditions should be met: (1) the asymptomatic infected person and the second-generation case have a clear contact history, and the second-generation case, after his/her onset of illness, has no history of contact with the asymptomatic infected person; (2) the onset of the second-generation case happened within 14 days after the last contact with the asymptomatic infected person; (3) the second-generation case had no other relevant exposure history or contact history than with the asymptomatic infected person.

It is recommended that sampling be done as early as possible during investigation. If sampling in the case of positive specimens of the asymptomatic infected person was earlier than the onset of the second-generation case, the evidence is considered to be stronger. In addition, it is recommended to collect serum specimens on the day of investigation and again 3-4 weeks later for future reference.

6. Analysis of transmission routes.

During field investigations, make sure the following information is collected: type of contact, contact distance, length of contact time, personal protection during contact, hand hygiene and other relevant conditions. Investigate the floor area of the exposure site, density of people, and the ventilation and air conditioning used to comprehensively analyze possible routes of transmission.

For a cluster of cases related to a closed space such as an airplane, a carriage in a high-speed train, an Internet bar, or a karaoke bar, analyze the correlation between development of illnesses of individuals and the index case in terms of seat distance, duration of short-distance conversation, toilet exposure, hand hygiene, and personal protection. If the time and spatial distribution of cases cannot be explained by droplet transmission or contact transmission and the possibility of aerosol transmission is suspected, it is recommended to collect air samples and environmental smears and swabs from the cabin, high-speed train carriage, toilet, or other relevant places to test the virus content and activity.

\section{Report outline}

\section{Background}

Describe the process of event detection and the general situation of the local outbreak including the number of cases, deaths, and case fatality rate.

2. Epidemiological investigation

(1) Describe the total number and classification of cases (including confirmed cases, suspected cases and asymptomatic infected persons), severe cases, and deaths.

(2) Describe each case by onset date including the basic information (name, age, gender, occupation, residential address at the time of onset, and ID number); progression of symptoms, diagnosis, and treatment; clinical manifestations; specimen collection and testing; progression and outcome of disease; exposure history; close contacts; tracking of activities after onset; and personal protective measures.

(3) According to the case investigation results, draw the epidemic curve, time sequence diagram, and case relationship diagram, then organize and summarize the key information of the cluster investigation and complete the form shown in Table S1.

\section{Investigation of place of exposure}

Describe the environment of the exposure place, the number of people with a common exposure, personal contact and protection measures. If necessary, draw a diagram of the exposed area.

4. Investigation of close contacts 
Describe the relationship between the case and his/her close contacts, type and frequency of contact and the first and last contact time; determine the total number of close contacts, their outcomes, and the number of people involved.

5. Measures taken

Describe the type, time, and implementation of the prevention and control measures for the cluster.

6. Investigation findings

Determine the generation of cases in the cluster, chains of transmission, and the source and route of transmission.

7. Recommendations

On the basis of the investigation findings and problems identified, propose targeted prevention and control recommendations. 


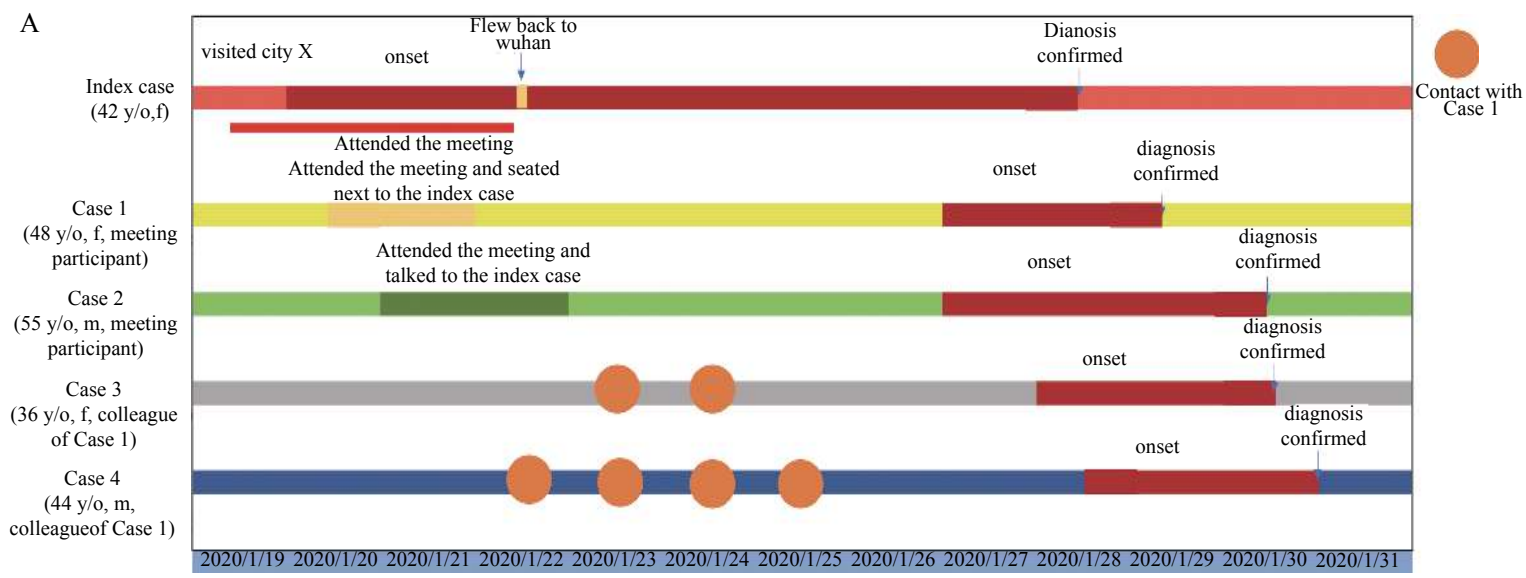

University student from Wuhan;

B

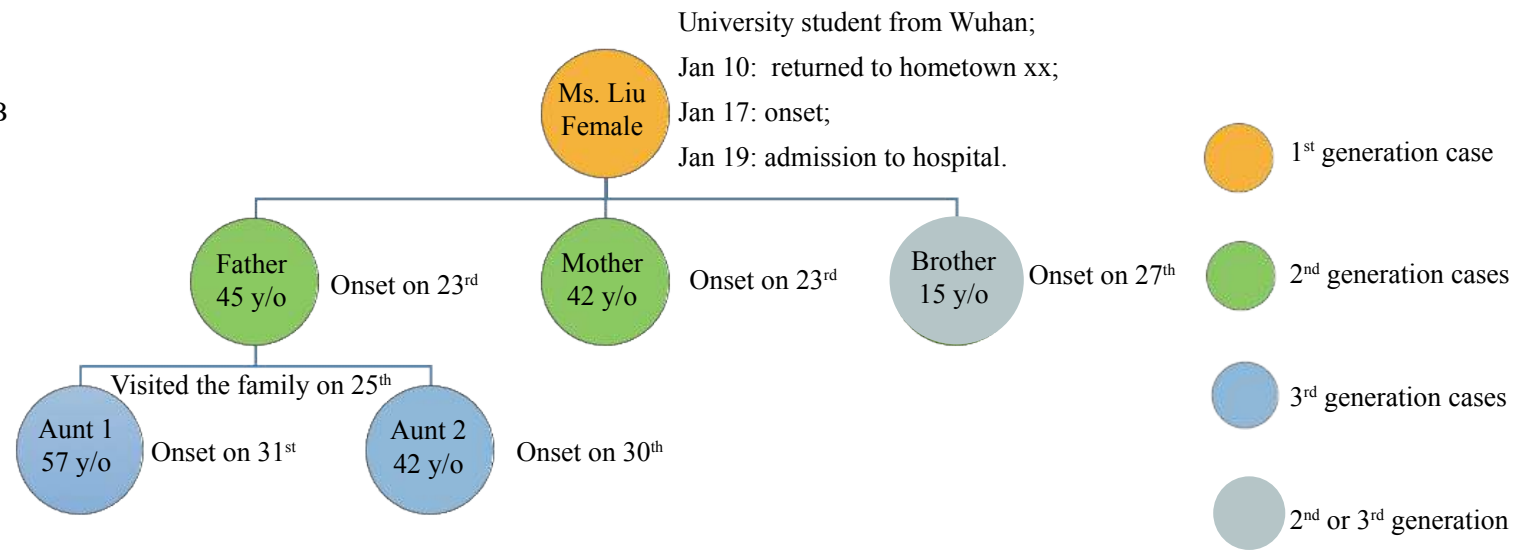

SUPPLEMENTARY FIGURE S1. Sample diagrams of a cluster outbreak. (A) Temporal sequence of meeting/business clusters. (B) Relations in a family cluster. y/o=years old. 


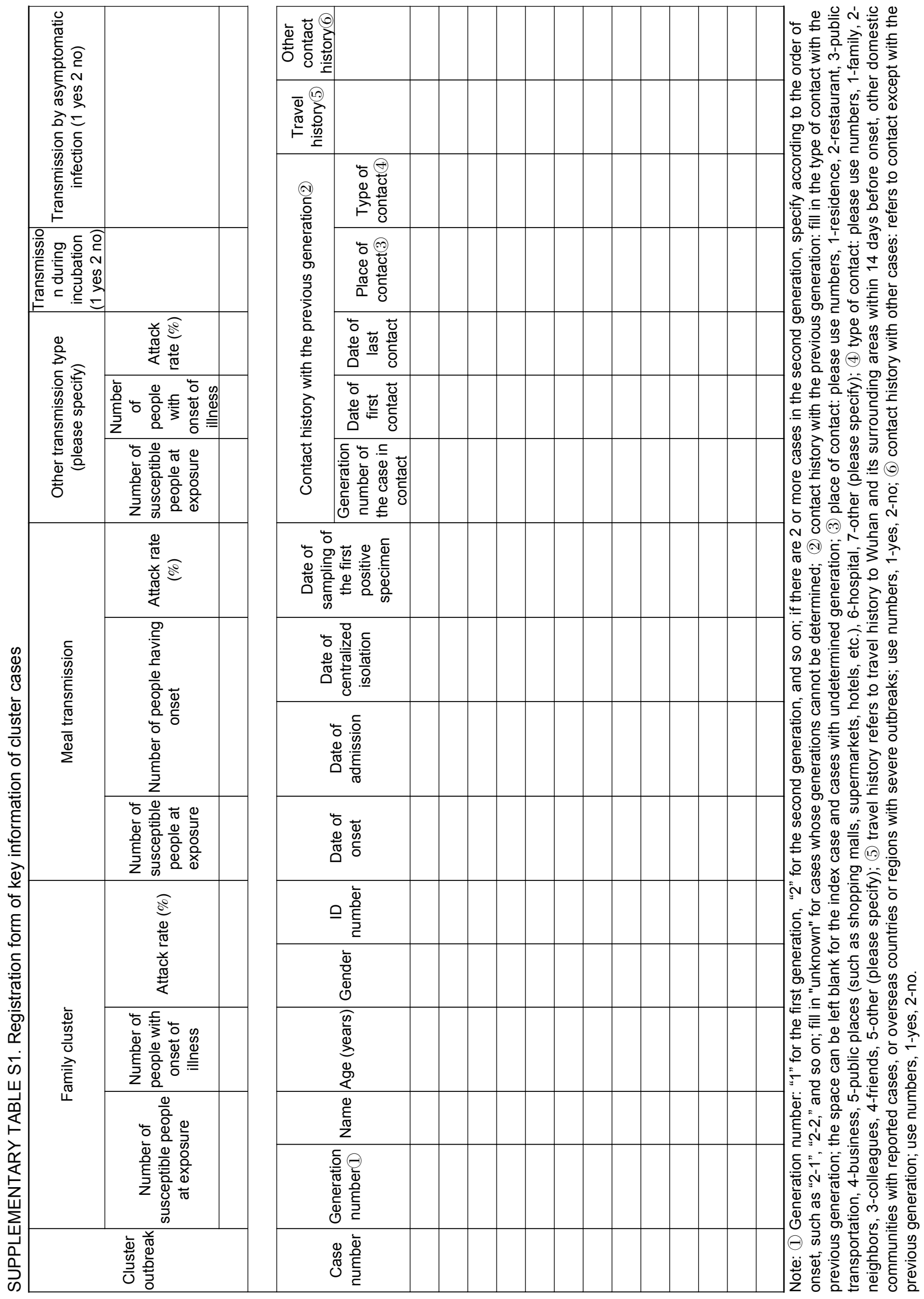

